\title{
Una mirada a la protección contractual del consumidor en Italia*
}

\section{Stefano Pagliantini ${ }^{* \star}$}

Resumen: El escrito examina la protección contractual del consumidor en el ordenamiento jurídico italiano, considerando también la influencia de las sentencias del Tribunal de Justicia Europeo. En particular, se exponen los rasgos distintivos de la disciplina de protección del consumidor y se valoran las principales proyecciones sistemáticas de esta disciplina en relación con la disciplina general del contrato.

Palabras clave: consumidor, contratos del consumidor, deber de información, transparencia, cláusulas abusivas, principio de efectividad.

\section{A brief look at the contractual consumer protection in Italy}

Aвsтract: The paper deals with the protection of consumers in the Italian legal system also considering the influence of the decisions from the European Court of Justice. In particular it deppens into the distinctive features of consumer pro-

El presente escrito corresponde a la voz "Consumidor (tutela del)", de la Enciclopedia Treccani, en prensa.

Trad. del italiano: Indira Díaz Lindao.

Fecha de recepción: 22 de diciembre de 20I4. Fecha de aceptación: I6 de marzo de 2015.

Para citar el artículo: S. Pagliantini. "Una mirada a la protección contractual del consumidor en Italia", Revista de Derecho Privado, Universidad Externado de Colombia, n. ${ }^{\circ} 28$, enero-junio de 20I5, pp. I9I-200. DOI: I0.1860I/or234366.n28.07

* Doctor en Derecho por la Università degli Studi di Pisa. Profesor ordinario de Derecho Privado de la Universidad de Siena, Italia. Contacto: stefano.pagliantini@unisi.it 
tection Law and highlights the special systemic proyections in comparisson with the general Law of Contracts.

Keywords: Consumer, consumer contracts, duty of disclosure, transparency, unfair contract terms, principle of effectiveness.

La definición legal de consumidor, sin perjuicio de una plurivalencia semántica del término que se presta a una variedad de usos y versiones, se encuentra en el artículo 3, literal a), del código del consumo italiano (en adelante cod. cons.), según el cual, es tal "la persona física que actúa con fines ajenos a la actividad empresarial o profesional que eventualmente desarrolla”. Su opuesto, con el fin de encuadrar con suficiente nitidez la figura de los contratos del consumidor, es el profesional, esto es, la "persona física o jurídica que actúa en el ejercicio de la propia actividad empresarial o profesional, o su intermediario" (art. 3 lit. c, cod. cons.).

A efectos de garantizar la libre competencia en el mercado que promueve la Unión Europea por medio del sistema de protección del consumidor, en el curso de las últimas tres décadas y en aplicación de los artículos 38 de la Carta de Derechos Fundamentales de la Unión Europea (CDFue) y i69 del Tratado de Funcionamiento de la Unión Europea (TFUE), el legislador europeo ha contribuido a la creación de un complejo orden jurídico de protección del consumidor que contrarresta la situación de asimetría (informativa) y de dependencia (económica) endémicamente existente cuando estos actúan en el mercado para la adquisición de bienes y servicios.

Bajo la concepción ideológica de que es la maximización de los consumos individuales la que trae consigo un mercado eficiente -comoquiera que es el mercado de consumo y no otro el llamado a crear el mayor bienestar colectivo-, la Unión Europea ha dispuesto un aparato normativo de tutela del consumidor cuya esencia en concreto no parece orientada a la finalidad de proteger los valores de la persona, sino que, por el contrario, se presenta objetivamente inmersa en una lógica gobernada por las dinámicas de un mercado competitivo que se pretende ex lege único.

La perspicacia de IRTI y, recientemente, las observaciones de las sentencias de las Sezione Unite de la Corte de Casación italiana (26242/2014) capturan vívidamente la sustancia del fenómeno: "tutelar al consumidor significa proteger el sistema de la competencia". Por tanto, es un modelo de tutela que "no se desarrolla en contra, sino dentro de la lógica capitalista" ${ }^{\mathrm{I}}$.

De otro lado, más allá de la circunstancia histórica de que ya la experiencia hegeliana conocía una vívida fundación de este derecho, se debe resaltar que la armonización europea ha tenido lugar a través de directivas, generalmente

I N. Irti, La fondazione begeliana del diritto dei consumatori, en P. Sirena (coord.), Il diritto europeo dei contratti d'impresa, Milano, 20o6, p. 479. 
concebidas de dos formas: como de armonización mínima (en cuanto sus disposiciones son inderogables in peius por cada uno de los países de la Unión); o de armonización máxima (en cuanto sus disposiciones son obligatorias e inderogables incluso in melius), y sin desdeñar una modalidad intermedia llamada de armonización ciblée o "pretendida".

En fin, los mecanismos de tutela del consumidor corresponden a una lógica de protección privatista, de carácter individual y colectivo (arts. I 39 y I 40 cod. cons.), a la cual se está sumando cada vez más una protección pública (public enforcement), sintetizada en un conjunto de sanciones pecuniarias de carácter público y remitidas principalmente a la competencia de la Autoridad Garante de la Competencia y del Mercado (arts. 27, párr. I bis y 66 cod. cons.).

En conclusión, estamos ante un sistema mixto y en evolución, caracterizado por un alto grado de volatilidad que, en consecuencia, dificulta la confiabilidad de su análisis unitario.

Se trata de un sistema ubicado dentro del Código de Consumo (D. Lgs. 2006/2005) y consiste en una serie de preceptos que derogan o integran las reglas ordinarias contenidas en el Código Civil italiano (art. I469-bis c.c.) en sentido más favorable al consumidor; este estatuto de protección del consumidor se resume en un catálogo de derechos que pueden ser presentados expositivamente en el siguiente orden progresivo:

a) una información precontractual obligatoria (arts. 48 y 49 cod. cons.), inmodificable unilateralmente y que a veces constituye parte integrante del contrato, de donde se deriva su operatividad incluso en ausencia y no solo en sustitución de las cláusulas que le son contrarias (arts. 49, párr. 5, y 72, párr. 4);

b) un registro documental de la información precontractual, ya sea en medio físico o electrónico, confiado a formas modelo que se exigen conjuntamente, pero cada vez más en forma separada del (eventual) formalismo del acto (arts. 72, párr. I, cod. cons. у I I 7 del Texto Único Bancario, en adelante тUв). Se trata, al menos normalmente, de formalidades en secuencia (arts. 50 y 5 I cod. cons.), reagrupables en torno al concepto de Vetragsanbahnung, es decir, a la celebración de un contrato en vista de una decisión negocial realmente informada y consciente;

c) un control ex fide bona de los contratos de adhesión con el fin de neutralizar la inserción de cláusulas conocidas como vejatorias, definidas precisamente como aquellas que causan, en contra de la buena fe, un desequilibrio significativo entre los derechos y obligaciones del contrato en perjuicio del consumidor (arts. 33 a 37 cod. cons.);

d) una transparencia del reglamento contractual orientada a la finalidad de un consumo eficiente; que se encuentra asistida por el canon de la interpretación más favorable al consumidor (art. 35, párr. 2, cod. cons.), y que comporta, en el caso de que la falta de transparencia ataque directamente las cláusulas económicas del contrato, un control (por reflejo) del carácter abusivo de las mismas (art. 
34, párr. 2, cod. cons. y sentencia del Tribunal de Justicia de la Unión Europea, en adelante stjue, caso Caja de Ahorros, C-484/o8);

e) una nulidad de protección de doble perfil, por ser puesta al servicio de las reglas del mercado (Cass. 26242/20I4), caracterizada por su parcialidad siempre que sea posible, en el sentido de que la nulidad de una cláusula no debe conducir a la nulidad de todo el negocio, a menos que el mismo no pueda sobrevivir sin ella (art. 36, párr. I-3 cod. cons.; stjue, casos Pereničová y Perenic, C-453/ro), y de reconocimiento oficioso en la medida en que beneficie al consumidor, de tal manera que en el curso de un proceso el consumidor podrá siempre oponerse a la declaración de nulidad de la cláusula si considera que su aplicación le favorece;

f) un derecho de retracto para remediar el efecto sorpresa de algunas ventas agresivas ${ }^{2}$ o de aquellas en las que el consumidor no tiene la posibilidad de probar el producto al momento de adquirirlo; retracto generalmente concebido como una terminación unilateral y ad nutum, que normalmente no está sometida a ninguna formalidad (excluida en todo caso la posibilidad de hacerlo efectivo a través de la conducta concluyente del consumidor); y que puede ser ejercido dentro del término establecido por la ley (dies ad quem), término que es prorrogable en el tiempo en el evento en que el profesional no proceda a informar al consumidor, en todo o en parte, de su derecho de retracto (arts. 52 y 72 cod. cons.). En todo caso, se trata de una prórroga que no actúa de manera indefinida (sine die) (stjue, caso Heininger, C-48I/99), sino dentro de un término máximo que coincide con el hecho del cumplimiento integral y satisfactorio de la lex contractus (stJue, caso Hamilton, C-41 2/06).

Como reflejo de la distinción entre los search y los credence goods 3 , y solamente en el área de las operaciones de crédito, el derecho de retracto admite además una postergación de su término legal (I 4 días), el cual solo comenzará a correr a partir del momento en el cual el consumidor haya recibido toda la información y

2 Nota de la trad.: En el derecho italiano y más en general en el derecho comunitario europeo, la denominación ventas "agresivas", propia de la terminología del derecho de consumo, es atribuida a las "ventas realizadas por fuera de los locales comerciales" y las "ventas a distancia", para las cuales el legislador ha previsto unos mecanismos especiales de protección contractual del consumidor (arts. 48 a 59 cod. cons.). De la misma manera sucede en Colombia, en donde este tipo de ventas han sido denominados legalmente como "ventas con utilización de métodos no tradicionales" y "ventas a distancia" (art. 4, nums. I4 y I 5 Ley I480/20I I), también con la introducción de algunas medidas especiales de protección del consumidor (arts. 46 a 48 Ley I480/20 I I).

3 Nota de la trad.: Estos términos corresponden a las categorías a través de las cuales son clasificados los servicios y bienes de consumo dependiendo del momento en el cual los consumidores están en capacidad de valorar su calidad. En este sentido se distingue entre los "search goods", como bienes o servicios que pueden ser valorados fácilmente por los consumidores antes de ser adquiridos; los "experience goods", aquellos cuya cualidad puede ser valorada por los consumidores solo con posterioridad a su adquisición; y los "credence goods", aquellos bienes o servicios cuya cualidad no puede ser valorada fácilmente por los consumidores, aun pasado un tiempo de su adquisición. 
las condiciones de ley sobre el contrato (arts. I 25-bis y I 25 ter тuв, y 67 duodecies, 1, b) cod. cons.);

g) el derecho a la entrega oportuna del bien comprado, acompañado de la facultad de resolver extrajudicialmente el contrato en caso de incumplimiento (o tardío cumplimiento) en la entrega por parte del profesional (art. 6r cod. cons.);

h) Como excepción al principio res perit domino, una regla especial sobre la transferencia del riesgo, que consiste en que el riesgo por la pérdida o por el daño de la cosa solo se transfiere al comprador en el momento en que este entra en posesión material del bien (art. 63 cod. cons.); y, superando la distinción clásica entre vicio material ${ }^{4}$ y defecto de calidad 5 , un derecho a la conformidad, en los contratos de venta de bienes de consumo, con una responsabilidad del profesional que comporta tanto remedios de conservación del contrato ${ }^{6}$ como remedios ablativos ${ }^{7}$, aunque no a elección del consumidor (arts. I 28 a I $35 \mathrm{cod}$. cons); finalmente,

i) una competencia judicial del consumidor, si es cierto que todas las controversias derivadas de la relación contractual celebrada por este último con un profesional son remitidas, como una excepción a las reglas generales de competencia territorial (arts. I 8 y 20 c.p.c.), a la competencia exclusiva del juez de su lugar de residencia o domicilio (arts. 33, 1, u) y 66 bis ibíd.). Es en todo caso una competencia unilateralmente inderogable, ya que el juez solo podrá declarar oficiosamente la nulidad de la cláusula que la modifica en el evento en que sea el profesional quien llame a juicio al consumidor en el (diferente) foro pactado en el contrato (Cass. I875/2012).

Finalmente, el esquema apenas expuesto se completa con la probibición general, impuesta a los profesionales por el artículo 20 cod. cons., de adoptar prácticas comerciales desleales en contra del consumidor, en la doble versión de prácticas engañosas o agresivas, en cuanto idóneas para distorsionar el comportamiento económico; prohibición que se encuentra reforzada por las dos listas negras de prácticas desleales contenidas en los artículos 23 y 26 cod. cons.

Ahora, si bien es a través de los mecanismos de tutela descritos supra, literales a)-i), que el catálogo de los llamados derechos fundamentales de los consumidores (consagrados en el art. 2, lits. a-g, cod. cons.) adquiere un cuerpo más visible, es por su carácter irrenunciable (previsto en el art. I43 cod.cons.) que los mismos se instituyen en instrumento de limitación de la libertad contractual del profe-

4 Nota de la trad.: Vicios existentes cuando el bien comprado presenta una anomalía material que lo hace inidóneo para su uso o disminuye en modo apreciable su valor.

$5 \quad$ Nota de la trad.: Se trata de la falta de las calidades prometidas o esenciales para el uso de la cosa objeto de la compraventa.

$6 \quad$ Nota de la trad.: Como la reparación o sustitución del bien, o la reducción adecuada del precio.

$7 \quad$ Nota de la trad.: Como la resolución del contrato. 
sional, y al mismo tiempo, de recalificación de la libertad del consumidor en el intento por hacer de él un actor racional del mercado.

Las sociedades de hoy, es cierto, "convirtiéndose en sociedades de los consumidores, podrían entenderse como [...] la venganza de Lilliput" : condición imprescindible, sin embargo, es que, en un contexto dominado por una contratación masiva que generalmente genera "pequeñas reclamaciones", exista una acción colectiva (art. I40-bis) y funcione como técnica de reglamentación indirecta del mercado.

De hecho, la última etapa recorrida por la disciplina de tutela del consumidor se enfoca en el plano de la "Materialisierung" de la tutela, entendida como el derecho de los consumidores a una protección que, de conformidad con lo dispuesto en el artículo 47 CDFUE, se caracterice por su efectividad.

En efecto, bajo la premisa de que la condición del consumidor es relevante solo en atención a su condición de sujeto vulnerable, porque su libertad para negociar es de tipo paramétrico y no estratégico, el remedio contractual utilizable de caso en caso es seleccionado con base en su nivel de disuasión, en el sentido de una primacía del mecanismo de tutela que más y de mejor manera pueda desincentivar aquel abuso del poder contractual del profesional que se concreta en trasladar al consumidor los costos de transacción de tal o cual operación económica.

Se trata de una efectividad orientada hacia el mercado (market oriented), sin perjuicio de la intención de construir una economía social del mercado (Marktwirtschaft soziale), que el Tribunal de Justicia de la Unión Europea concibe, actualmente, según un esquema compuesto por:

a) la primacía de una tutela específica respecto a la resarcitoria, especialmente cuando están en juego derechos fundamentales como el de vivienda, u otras situaciones jurídicas de rango primario (en este sentido se destacan, principalmente, stjue, casos Aziz, C-4I 5/I I, Kušionová, C-34/I 3 y Sánchez Morcillo, C-I69/I4);

b) la pérdida total y no parcial del derecho a los intereses, cuando el comerciante haya desatendido una correcta valoración de la solvencia crediticia del consumidor mutuario (stjue, caso Le Crédit Lyonnais SA, C-565/I 2), en vista de una responsabilidad objetiva (strict liability) de la empresa que actúa de manera incorrecta;

c) la integración dispositiva del contrato cuando, excluida la cláusula abusiva, este no es autosuficiente para regular la relación contractual (sTJUE, caso Kasler Arpád, C-26/I3), con lo cual se abre paso a un proceso de transfiguración de las normas dispositivas, que si bien no da lugar a su conversión obligatoria, sí las impregna de un matiz cripto-imperativo. 
De manera especial, lo que se delinea es una exaltación de las normas dispositivas como modelo ideal de reglamentación, a fin de detener aquella inobservancia del derecho dispositivo que se traduce en la imposición sistemática de un contenido contractual abusivo por parte del profesional. Sin embargo, se trata de un resultado que se obtiene ex post, esto es, luego de una difusa praxis interpretativa que se abstiene de derogar las normas dispositivas en los eventos en que, excluida la cláusula abusiva, el contrato puede mantenerse.

d) la legitimidad de la cláusula de ius variandi por parte del profesional, a condición de que esta sea transparente y siempre que la naturaleza competitiva del mercado de referencia sea tal que asegure la terminación unilateral del contrato por parte del consumidor, en forma auténtica y no virtual (sTJUE, caso RWE Vertrieb, C-92/I I); finalmente,

e) la capacidad de reducción oficiosa del precio, en caso de que el vicio del cual adolece el producto vendido sea de leve entidad, donde el quanti minoris se muestre como el único remedio accionable para satisfacer el derecho del consumidor a la conformidad del bien (stjue, caso Duarte Hueros, C-32/ I 2).

En este panorama en movimiento no faltan los casos en los cuales el canon vinculante de la interpretación conforme termina por acuñar la forma de una mixtura remedial propiamente dicha (stjue, casos Pannon GSM Zrt., C- 243/o8; Invitel, C-472/Io, y Banif Plus Bank, C-472/I I): sin embargo, dejando a salvo las diferencias específicas que caracterizan cada caso en particular, todo parece estar encauzado por la idea de aplicar el remedio que en mejor manera pueda erigirse en una especie de arma disuasoria (Abschrekungseffekt) del profesional, cual factor de optimización de la tutela del consumidor, a veces, incluso, prescindiendo de un principio de razonabilidad (stjue, casos Weber/Putz, C-65/o9 y C-87/o9, y Messner, C-489/07) en la ponderación de los intereses contrapuestos.

Restan dos cuestiones en este breve recorrido de conjunto: la primera, cómo opera la relación entre la disciplina de protección del consumidor y la disciplina del derecho común, dado el canon de armonización selectiva que se orienta a privilegiar la disposición más favorable al consumidor (art. I469-bis c.c.); y la segunda, hasta qué punto sigue siendo vinculante para el intérprete la noción pura de consumidor citada al inicio de este escrito, la cual, si bien permite delimitar con suficiente precisión el perímetro de los contratos asimétricos celebrados entre un profesional y un consumidor (contratos también denominados $\mathrm{B}_{2} \mathrm{C}^{9}$ ), no permite, viceversa, que la disciplina orientada especialmente a proteger la posición del consumidor contribuya al mismo tiempo a proteger otras formas de debilidad contractual, en particular, la de las pequeñas y medianas empresas (PYME).

9 Nota de la trad.: Acrónimo que indica la expresión Business to Consumer y que generalmente se contrapone al acrónimo $\mathrm{B}_{2} \mathrm{~B}$ que indica la expresión Business to Business. 
Ahora bien, en cuanto a la primera cuestión, si se pretende tomar en serio la distinción entre un primer derecho ${ }^{\mathrm{IO}} \mathrm{y}$ un segundo derecho ${ }^{\mathrm{II}}$, se debe necesariamente archivar la idea tradicional de una especialidad pura de la disciplina de protección del consumidor relevante a la luz de una ley especial.

En efecto, el canon de la norma más favorable al consumidor supone que las relaciones de consumo sean gobernadas por una previsión general del código civil, solo si y en la medida en que el intérprete haya determinado previamente la ausencia de otras disposiciones que puedan ser útilmente invocadas fuera del perímetro de los artículos I32 I y siguientes ${ }^{\mathrm{I} 2}$, disposiciones que, aplicando la técnica de producción de normas a través de normas, bien podrían considerarse inherentes al mismo código de consumo u otros subsistemas normativos de carácter sectorial.

Quien en cambio aboga, en el caso de una "laguna" en la regulación de los contratos de consumo, por la aplicación inmediata de la disciplina del código civil, parte más o menos conscientemente de la premisa de que las normas del derecho de los consumidores deben interpretarse siempre en forma restrictiva, y por eso mismo no son susceptibles de una aplicación directa o analógica al interior de su perímetro como subsistema normativo. Este razonamiento tiene, sin embargo, un defecto, ya que termina por fundarse exclusivamente en lo dispuesto en el artículo 38 cod. cons. ${ }^{13}$, casi como si el sistema de la legislación de consumo fuera un conjunto de normas que contraviene las reglas generales, en el sentido estricto previsto en el artículo I4 de las disposiciones generales del derecho (preleggi) ${ }^{\mathrm{I} 4}$, olvidando entonces que el derecho del consumo está igualmente gobernado por normas no menos generales que las del codice civile (arts. 36 y 48 cod. cons.).

Eventualmente, el verdadero problema podría radicar en el canon de la armonización máxima o completa, ya que la prohibición de realizar una derogación in melius de la normativa comunitaria de protección del consumidor a efectos evitar la configuración de regímenes de tutela divergentes entre los diferentes Estados, podría conducir a que el conjunto de las materias gobernadas por este

Io Nota de la trad.: Se alude a la disciplina del derecho común de los contratos concebida para las relaciones contractuales entre partes en pie de igualdad y a la cual se hace referencia supra en el texto.

I I Nota de la trad.: Se alude a los contratos del consumidor como disciplina normativa de carácter especial y autónoma.

I Nota de la trad.: Los artículos I32 I y siguientes del codice civile corresponden a las disposiciones preliminares de los contratos en general, inmersas en el libro IV del código denominado "de las obligaciones".

I 3 Nota de la trad.: El artículo 38 cod. cons. establece expresamente: "En lo no previsto por el presente código, a los contratos celebrados entre el consumidor y el profesional se aplican las disposiciones del código civil”.

I4 Nota de la trad.: El artículo I4 de las disposiciones generales del cod. cons. prevé: "Las leyes penales y las que son excepción a las reglas generales u otras leyes no se aplican fuera de los casos y de los tiempos en ellas considerados". 
tipo de armonización termine por configurarse como un articulado autosuficiente y que tendencialmente se autointegra.

Respecto a la segunda pregunta, en cambio, una novedad parece vislumbrarse en el Considerando i 7 de la Directiva 20 I/83/UE, la cual toma distancia de la jurisprudencia consolidada del Tribunal de Justicia de la Unión Europea (stjue, caso Gruber, C-464/or), y califica como contratos del consumidor aquellos celebrados con propósitos mixtos, siempre y cuando no predomine el propósito comercial; adicionalmente, desde el punto de vista legislativo ya floreció la protección contractual de los contratantes débiles que no se encuadran dentro de la categoría del consumidor, cuanto menos, de las microempresas (arts. I 8 d-bis) cod. cons. у г I 8 тив).

En el estado actual, sin embargo, resulta difícil conceptualizar una summa divisio en la que el contrato de derecho común, celebrado entre partes en pie de igualdad, sea concebido como una categoría contrapuesta a la de los contratos asimétricos como esquema arquetípico al cual se puedan reconducir todos los tipos de contratante débil. Así las cosas, el modelo italiano permanece híbrido frente al monismo matizado por el derecho alemán ${ }^{15}$ (\$305 вGв) у frente a la bifurcación francesa (arts. I 32 - I Cod. consomm. y 442-6 Cod. comm.) ${ }^{\mathrm{I} 6}$, en virtud de la individualización de una tercera categoría denominada terzo contratto $^{17}$, relevante, en todo caso, más como una intuición problemática que como una categoría ordenadora.

\section{Bibliografía}

Carbonnier, J. Flexible droit, Paris, I9927, trad. al italiano, Milano, i997.

Castronovo, C. Diritto privato generale e diritti secondi. La ripresa di un tema, en Eur. dir. priv., 2006, pp. 397 ss.

D’Amico, G. L'abuso dell'autonomia negoziale nei contratti con i consumatori, en P. SIRENA (coord.), Il diritto europeo dei contratti d'impresa, Milano, 2006, pp. 32 I ss.

I 5 Nota de la trad.: El cual regula unitariamente la tutela de los contratos celebrados con asimetría de poder contractual de las partes, sean estos celebrados entre un "consumidor" y un "profesional", o entre "profesionales", aunque con algunos matices entre ellos, ya que para los primeros se establecen mecanismos adicionales de protección contractual de la parte débil.

i6 Nota de la trad.: Los cuales regulan, por una parte, los contratos del consumidor, y por otra, las reglas de la competencia aplicables a los contratos entre empresarios.

I 7 Nota de la trad.: Se trata de una categoría dogmática que se orienta a diferenciar entre: los contratos de derecho común caracterizados por la igualdad de fuerza contractual entre las partes; los asimétricos celebrados entre un "consumidor" y un "profesional", y el "terzo contratto", al cual se reconducen, fundamentalmente, las hipótesis de negociación asimétrica entre profesionales o empresarios, bajo el presupuesto de que a las mismas se debe aplicar un paradigma normativo de protección del contratante débil distinto al de los contratos del consumidor. 
Mazzamuto, S. Il contratto di diritto europeo, Torino, 2012.

Irti, N. La fondazione hegeliana del diritto dei consumatori, en P. SiRENA (coord.), Il diritto europeo dei contratti d'impresa, Milano, 2006, pp. 475 ss.

Vettoni, G. (al cuidado de), Il contratto dei consumatori, dei turisti, dei clienti, degli investitori e delle imprese deboli. Oltre il consumatore, Padova, 2013. 